\title{
Monitoring and Diagnosing Neonatal Seizures by Video Signal Processing
}

\author{
G.M. Kouamou Ntonfo \\ Department of Information Engineering, University of Parma, Italy \\ Advisor/s: Riccardo Raheli \\ Date and location of PhD thesis defense: 4 March 2013, University of Parma
}

Received 10th Feb 2014; accepted 28th May 2014

\begin{abstract}
Clinical operators in one of the most difficult health care fields, namely neonatal neurology, on a daily basis have to face the diagnosis of epileptic seizures. Most of the neonates affected by perinatal diseases are at risk of neonatal seizures, which are the most common sign of acute neurological dysfunctions and must be promptly and accurately recognized in order to establish timely treatments. Traditional diagnostic methods are based on ElectroEncephaloGraphic (EEG) monitoring. The neonatal EEG analysis is, however, a very specialistic and time-consuming technique which requires particular skills not always easily available in Neonatal Intensive Care Units (NICUs). Therefore, non-invasive, real-time, automated, low-cost, wide-scale diagnostic methods and equipments capable of reliably recognizing neonatal seizures would be of significant value in the NICUs.

Whilst the importance of promptly diagnosing the presence of neonatal seizures is clear, there are no actual methods to early recognize or detect such pathological behaviors, nor currently available instruments to predict them. The only available and reliable method is the EEG, which is moderately invasive and needs well-trained medical personnel to be correctly administered and interpreted.

A very appealing alternative, with respect to the EEG, to automatically detect the presence of seizures consists in acquiring, through a video camera, the movements of the newborn's body and properly processing the relevant video signal. The goal of an effective image processing algorithm is the detection of "unusual" movements of the newborn. The aim of automatic detection and classification of neonatal seizures through a video camera is not to completely replace the EEG (still required for accurate diagnosis), but to make a realtime, low-cost, preliminary, automatic diagnosis based on clinical aspects of neonatal seizures. In other words, an automatic video camera-based system could be used to permanently monitor every patient in the neonatal care unit, whereas the EEG would be required for a definitive diagnosis only when the system indicates, with high probability, the potential presence of seizures. For this purpose several approaches, developed at the Department of Information Engineering, in collaboration with the Department of Neurosciences, both of the University of Parma (Italy), have been proposed: periodicity-based, classification-based and clustering-based approaches.
\end{abstract}

In periodicity based approaches we first investigated on the presence of clonic and some subtle seizures using a single Red-Green-Blue (RGB) videocamera [1] [2]. We extracted, through proper low-complexity filtering,

Correspondence to: < gkntonfo@gmail.com>

Recommended for acceptance by $<$ Alicia Fornés and Volkmar Frinken $>$

ELCVIA ISSN:1577-5097

Published by Computer Vision Center / Universitat Autonoma de Barcelona, Barcelona, Spain 
a motion signal representative of the body movements of the newborn. In the presence of seizures, the motion signal shows a periodicity very similar to that of the body movements. By applying a periodicity detection algorithm, the periodicity is detected per window (i.e., over a sequence of video frames). Single window processing and multiple interlaced window processing are considered. While in the case of single windows the sensitivity is significantly high, the use of interlaced windows guarantees good values of specificity [3]. A significant drawback that we faced by using a single videocamera was the fact that the patient was filmed only from one viewpoint. Therefore, movements with a direction perpendicular to the videocamera plane was not accurately detected. In order to have a better coverage of the scene, we proposed another periodicity-based approach, based on the use of multiple videocameras and the estimate of a common periodicity feature of the extracted motion signals [4]. Precisely, two cases of multiple videocameras are studied: two RGB videocameras physically placed so that their visual fields intersect and the whole scene is covered and an RGB-Depth (RGBD) videocamera which provides in addition to an RGB image, a depth map of the target scene by a depth sensor. From preliminary results, we observed that this approach may lead to improvents of the detection performance for clonic and subtle seizures.

The classification based-approach relies upon the observation that myoclonic seizures are brief, rapid, single or arrhythmic repetitive jerks. Therefore, we make use of relevant video signal features for the diagnosis of neonatal myoclonic seizures [5]. In particular, a region of interest (ROI) is selected by identifying an area around the point where the maximum amplitude of the optical flow vector is observed. The motion signal of the ROI is extracted and the broadening factor and the maximum distance between consecutive pairs of zeros of the motion signal are used on a properly trained K-Nearest Neighbors classification algorithm in order to detect myoclonic seizures. This approach may also differentiate clonic and myoclonic seizures from random movements.

Finally we proposed a clustering-based approach [6] which is based on the observation that clonic and subtle seizures are characterized by repetitive movements. Consequently, the trajectories of pathological gestures for a newborn affected by seizures are similar and, therefore, recognizable. Observing that, during a seizure, the part of the body that moves faster is likely to be the one affected by pathological movements, the relevant moving limb is then tracked using a combination of template matching and optical flow algorithms. The idea is to use relevant motion trajectory features for gesture recognition. After a predetermined monitoring time, all relevant motion trajectories are clustered using the DBSCAN algorithm. Preliminary results show that the proposed approach allows to accurately detect neonatal clonic and subtle seizures.

\section{References}

[1] G. Ferrari, G.M. Kouamou Ntonfo, C. Copioli, R. Raheli and F. Pisani, "Low-Complexity Image Processing for Real-Time Detection of Neonatal Clonic Seizures", IEEE International Symposium on Applied Sciences in Biomedical and Communication Technologies (ISABEL 2010), 16(3):375-382, November 2010.

[2] G.M. Kouamou Ntonfo, G. Ferrari, R. Raheli and F. Pisani, "Low-Complexity Image Processing for Real-Time Detection of Neonatal Clonic Seizures", IEEE Transactions on Information Technology in Biomedicine (TITB), 16(3):375-382, 2012.

[3] F. Pisani, C. Spagnoli, E. Pavlidis, C. Facini, G.M. Kouamou Ntonfo, G. Ferrari and R. Raheli, "Real-Time Automated Detection of Clonic Seizures in Newborns", Clinical Neurophysiology, February 2014.

[4] L. Cattani, G.M. Kouamou Ntonfo, F. Lofino, G. Ferrari, R. Raheli and F. Pisani, "Maximum-Likelihood Detection of Neonatal Clonic Seizures by Video Image Processing", International Symposium on Medical Information and Communication Technology (ISMICT 2014), Florence, Italy, April 2014. 
[5] G.M. Kouamou Ntonfo, G. Ferrari, F. Lofino, R. Raheli and F. Pisani, "Extraction of video features for real-time detection of neonatal seizures", IEEE International Symposium on a World of Wireless, Mobile and Multimedia Networks (WOWMOM 2011), Lucca, Italy, 1-6, June 2011.

[6] G.M. Kouamou Ntonfo, G. Ferrari, F. Lofino, R. Raheli and F. Pisani, "Video processing-based detection of neonatal seizures by trajectory features clustering", IEEE International Conference on Communications (ICC 2012), Ottawa, Canada, 3456-3460, June 2012. 